\title{
Research Article \\ Efficiency Analysis of Multiple-Transmitter Wireless Power Transfer Systems
}

\author{
Jin Zhang $\mathbb{D}^{1}$ and Fangfang Wang $\mathbb{D}^{2}$ \\ ${ }^{1}$ College of Electronic and Information Engineering, Jinling Institute of Technology, 99 Hongjing Avenue, Jiangning District, \\ Nanjing 211169, China \\ ${ }^{2}$ School of Electronic Science and Engineering, Nanjing University of Posts and Telecommunication, 66 Xinmofan Road, \\ Gulou District, Nanjing 210003, China
}

Correspondence should be addressed to Jin Zhang; jinzhang@jit.edu.cn

Received 3 February 2018; Revised 9 April 2018; Accepted 17 April 2018; Published 8 July 2018

Academic Editor: Giuseppina Monti

Copyright (C) 2018 Jin Zhang and Fangfang Wang. This is an open access article distributed under the Creative Commons Attribution License, which permits unrestricted use, distribution, and reproduction in any medium, provided the original work is properly cited.

\begin{abstract}
The range of the power transmission region can be effectively enlarged by a multiple-transmitter (TXs) and single-receiver (RX) magnetic wireless power transfer (WPT) system. However, the power transfer efficiency (PTE) of the WPT system with two TXs and a single RX has been intensively studied in the past few years. Therefore, a deep analysis of multipleTX WPT systems needs to be implemented urgently to achieve the highest PTE in a wider range of power transmission. This paper analyzes a general multiple-TX single-RX system in detail based on the circuit model. Two optimal conditions, in which the feeding amplitude ratios need be equal to the mutual inductance ratios and the optimal load should be connected to RX, are derived to obtain the highest PTE. In the experiment part, the feeding amplitude ratios for multiple TXs are implemented by a proposed lumped transformer for the first time. The use of the lumped transformer reduces the complexity compared with the use of multiple inverters to achieve predetermined feeding amplitude ratios. MultipleTX systems are designed and measured to validate the theoretical analysis. Experimental results agree very well with theoretical calculations.
\end{abstract}

\section{Introduction}

The research of magnetic-resonance coupling is particularly prized in the areas of wireless power transfer (WPT) since this research was published in the US journal Science in 2007 [1]. Many interesting fields on magnetic-resonance WPT have been studied in just the last 10 years alone [2-19].

In $[2,3]$, the circuit model and evaluation method for conventional and multiple-coil WPT systems are presented to discuss the frequency splitting properties. The frequency splitting results in degraded output power at the original frequency when the transfer distance is shortened. To deal with the power transfer degradation due to frequency splitting, the schemes of nonidentical transmitting and receiving coils, antiparallel resonant loop transmitter (TX), and mixed magnetic and electric coupling have been proposed to prevent frequency splitting via suppressing the coupling strength in references [4-6]. Methods of Kirchhoff's voltage law, band-pass filter theory, and coupled-mode theory are compared and discussed in $[7,8]$. Based on those methods, characteristic analysis of goal setting WPT systems are implemented in [9-19]. The systems consisting of multiple receivers (RXs) can be applied well to the scenario of a single charging platform. This scenario supplies power for various electronic devices; therefore, multiple-RX systems have acquired comprehensive and deep studies $[13,15,16]$. On the other hand, the multiple-TX cases are considered for adding gain over longer distances comparing with singleTX cases $[14,17,18]$. As the number of TXs increases, the gain of the system is enhanced over a larger region. In [14], the authors presented a two-TX system which is optimized by the distance between the two TXs. The investigation shows that the proposed two-TX system can generate a more stable output power in a broader space than the single-TX 
case. Reference [17] introduced a practical power line synchronization technique with regard to frequency and phase to synchronize all TXs. In [18], since two TXs and a single RX are located at an arbitrary position and rotation angle, the amplitude ratio and the phase difference of the signals of the two TXs are optimized to obtain the highest power transfer efficiency (PTE). However, in these studies $[14,17,18]$, the analysis model and available conclusions are limited to two-TX systems.

In this paper, the PTE of a multiple-TX WPT system is investigated. The analytical expression of PTE is derived. We observe that the relationship between the amplitude in each TX and the mutual inductance between the RX and any TX crucially determines the highest PTE. Based on the analysis, two experimental schemes of efficiencies versus the number of TXs and power transfer distance are implemented later in this paper.

\section{Theoretical Derivation of Transmission Efficiency}

The operating wavelengths are considerably larger than the sizes of power coils and the transmission distance. Thus, circuit theory can be used to analyze the model of the WPT system. Figure 1 illustrates the equivalent circuit of a representative WPT system with multiple TXs and a single RX. $\operatorname{TX}_{i}(i=1,2, \ldots, n)$ is the expression of the $i$ th TX. The mutual inductance between $\mathrm{TX}_{i}$ and $\mathrm{RX}$ is represented as $M_{i R} . L_{R}, C_{R}$, and $r_{R}$ are the self-inductance, compensation capacitor, and equivalent series resistance (ESR), respectively, of the RX. Similarly, $L_{i}, C_{i}$, and $r_{i}$ are the selfinductance, compensation capacitor, and ESR, respectively, of the $i$ th TX. The self-resonant frequency for each resonator is $\omega_{0}\left(\omega_{0}=1 / \sqrt{L_{i} C_{i}}=1 / \sqrt{L_{r} C_{r}}\right)$.

Based on Kirchhoff's voltage law (KVL), at operating frequency $\omega$, the electrical relationship of the multiple-TX WPT system shown in Figure 1 can be described as

$$
\begin{aligned}
{\left[\begin{array}{c}
V_{1} \\
V_{2} \\
\vdots \\
V_{n} \\
0
\end{array}\right]=} & {\left[\begin{array}{ccccc}
Z_{1} & j \omega M_{12} & \cdots & j \omega M_{1 n} & j \omega M_{1 R} \\
j \omega M_{12} & Z_{2} & \cdots & j \omega M_{2 n} & j \omega M_{2 R} \\
\vdots & \vdots & \vdots & \ddots & \vdots \\
j \omega M_{1 n} & j \omega M_{2 n} & \cdots & Z_{n} & j \omega M_{n R} \\
j \omega M_{1 R} & j \omega M_{2 R} & \cdots & j \omega M_{n R} & Z_{R}
\end{array}\right] } \\
& \times\left[\begin{array}{c}
I_{1} \\
I_{2} \\
\vdots \\
I_{n} \\
I_{R}
\end{array}\right],
\end{aligned}
$$

where $V_{i}$ and $I_{i}(i=1,2, \ldots, n)$ are peak-value phasors, and all of $V_{i}$ are in phase. $Z_{i}=r_{i}+j\left(\omega L_{i}-1 / \omega C_{i}\right)$ and $Z_{R}=$ $r_{R}+R_{L}+j\left(\omega L_{R}-1 / \omega C_{R}\right)$ are the impedances of each TX

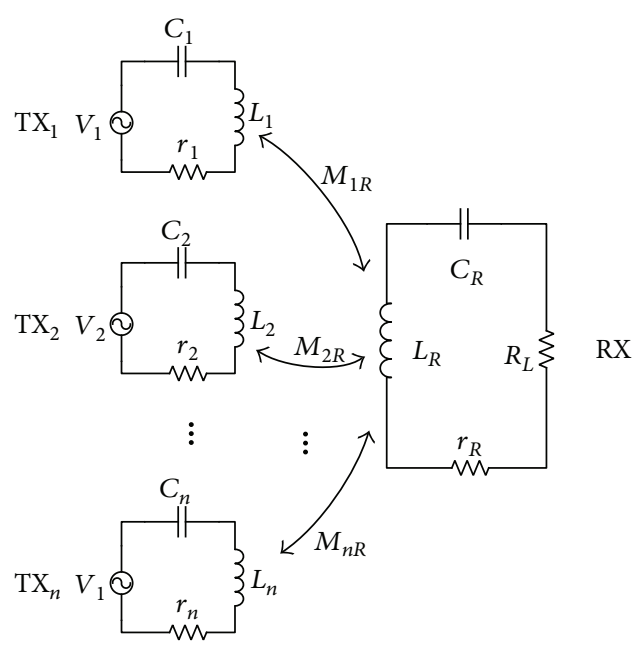

FIgURE 1: Circuit model of a multiple-TX single-RX WPT system.

and the single RX, respectively. In general, compared to the mutual inductance strengths between TXs and the RX $\left(M_{i R}\right)$, the ones between TXs $\left(M_{i j}, i, j=1,2, \ldots, n\right.$, and $\left.i \neq j\right)$ are small enough to be omitted, namely, $M_{i j}=0$. Therefore, under the resonance condition $\left(\omega L_{i}-1 /\left.\omega C_{i}\right|_{\omega=\omega_{0}}=0\right)$ and $M_{i j}=0$, formula (1) can be simplified to

$$
\begin{aligned}
{\left[\begin{array}{c}
V_{1} \\
V_{2} \\
\vdots \\
V_{n} \\
0
\end{array}\right]=\left[\begin{array}{ccccc}
r_{1} & 0 & \cdots & 0 & j \omega_{0} M_{1 R} \\
0 & r_{2} & \cdots & 0 & j \omega_{0} M_{2 R} \\
\vdots & \vdots & \vdots & \ddots & \vdots \\
0 & 0 & \cdots & r_{n} & j \omega_{0} M_{n R} \\
j \omega_{0} M_{1 R} & j \omega_{0} M_{2 R} & \cdots & j \omega_{0} M_{n R} & r_{R}+R_{L}
\end{array}\right] } \\
\times\left[\begin{array}{c}
I_{1} \\
I_{2} \\
\vdots \\
I_{n} \\
I_{R}
\end{array}\right] .
\end{aligned}
$$

Mesh currents $I_{i}$ and $I_{R}$ in $\mathrm{TX}_{i}$ and $\mathrm{RX}$ can be merged into two sets of (3) and (4) from (2):

$$
\begin{aligned}
V_{i} & =r_{i} I_{i}+j \omega_{0} M_{i R} I_{R}, \\
0 & =\sum_{i=1}^{n} j \omega_{0} M_{i R} I_{i}+\left(r_{R}+R_{L}\right) I_{R} .
\end{aligned}
$$

Solving (3), the formula for $I_{i}$ in terms of $I_{R}$ is

$$
I_{i}=\frac{V_{i}-j \omega_{0} M_{i R} I_{R}}{r_{i}} .
$$


Substituting (5) into (4) gets

$$
I_{R}=-\frac{\sum_{i=1}^{n}\left(j \omega_{0} M_{i R} V_{i} / r_{i}\right)}{\sum_{i=1}^{n}\left(\left(\omega_{0} M_{i R}\right)^{2} / r_{i}\right)+r_{R}+R_{L}}
$$

Solving simultaneous equations of (3) and (6), we obtain

$$
\frac{I_{i}}{I_{R}}=-\frac{V_{i}}{r_{i}} \times \frac{\sum_{t=1}^{n}\left(\left(\omega_{0} M_{t R}\right)^{2} / r_{t}\right)+r_{R}+R_{L}}{\sum_{t=1}^{n}\left(j \omega_{0} M_{t R} V_{t} / r_{t}\right)}-\frac{j \omega_{0} M_{i R}}{r_{i}}
$$

The PTE for the system is

$$
\begin{aligned}
\mathrm{PTE} & =\frac{R_{L}\left|I_{R}\right|^{2}}{\sum_{i=1}^{n} R_{i}\left|I_{i}\right|^{2}+\left(r_{R}+R_{L}\right)\left|I_{R}\right|^{2}} \\
& =\frac{R_{L}}{\sum_{i=1}^{n} R_{i}\left|I_{i} / I_{R}\right|^{2}+r_{R}+R_{L}},
\end{aligned}
$$

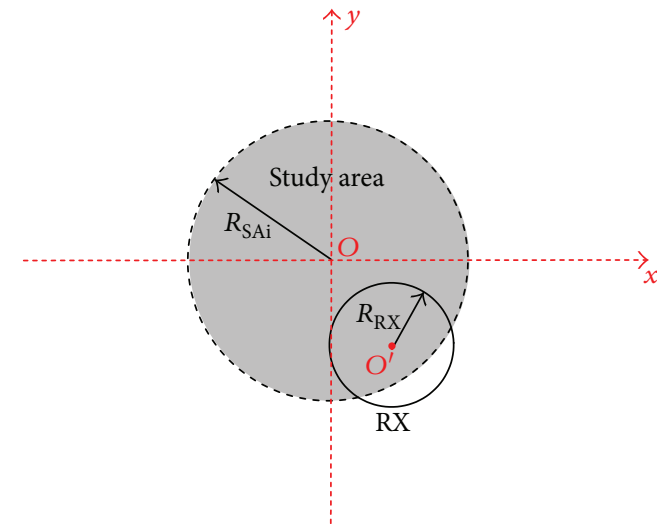

FIgURE 2: The RX moving within the circle of the study area for optimal PTE investigation.

where $I_{i}$ and $I_{R}$ are the currents flowing through the $i$ th TX and the load without considering radiation loss. Substituting (7) into (8), PTE can be represented as

$$
\mathrm{PTE}=\frac{R_{L}}{\sum_{i=1}^{n}\left\{r_{i}\left[\left(r_{R}+R_{L}\right) V_{i} / r_{i}+\omega_{0}^{2} \sum_{t \neq i}^{n}\left(M_{t R}\left(M_{t R} V_{i}-M_{i R} V_{t}\right) / r_{i} r_{t}\right)\right]^{2}\right\} /\left(\omega_{0} \sum_{i=1}^{n}\left(M_{i R} V_{i} / r_{i}\right)\right)^{2}+r_{R}+R_{L}} .
$$

It can be found from (9) that PTE achieves the optimal value, $\mathrm{PTE}_{\mathrm{OPT}}^{\prime}$, with fixed $R_{L}$ when $M_{t R} V_{i}-M_{i R} V_{t}=0$, namely,

$$
\frac{V_{i}}{V_{t}}=\frac{M_{i R}}{M_{t R}}
$$

Under condition of (10), we obtain

$$
\begin{aligned}
\mathrm{PTE}_{\mathrm{OPT}}^{\prime} & =\frac{R_{L} \omega_{0}^{2} \sum_{i=1}^{n}\left(M_{i R}^{2} / r_{i}\right)}{\left(r_{R}+R_{L}\right)\left(r_{R}+R_{L}+\omega_{0}^{2} \sum_{i=1}^{n}\left(M_{i R}^{2} / r_{i}\right)\right)} \\
& =\frac{\omega_{0}^{2} \sum_{i=1}^{n}\left(M_{i R}^{2} / r_{i}\right)}{R_{L}+r_{R}\left(r_{R}+\omega_{0}^{2} \sum_{i=1}^{n}\left(M_{i R}^{2} / r_{i}\right)\right) / R_{L}+2 r_{R}+\omega_{0}^{2} \sum_{i=1}^{n}\left(M_{i R}^{2} / r_{i}\right)} .
\end{aligned}
$$

From (11), the optimal load, $R_{L, \mathrm{OPT}}$, can be derived to obtain the final optimal efficiency, $\mathrm{PTE}_{\mathrm{OPT}}$, when $R_{L}=$ $r_{R}\left[r_{R}+\omega_{0}^{2} \sum_{i=1}^{n}\left(M_{i R}^{2} / r_{i}\right)\right] / R_{L}$.

$$
\begin{aligned}
R_{L, \mathrm{OPT}} & =r_{R} \sqrt{1+\frac{\omega_{0}^{2}}{r_{R}} \sum_{i=1}^{n} \frac{M_{i R}^{2}}{r_{i}}}, \\
\mathrm{PTE}_{\mathrm{OPT}} & =\frac{\sqrt{1+\left(\omega_{0}^{2} / r_{R}\right) \sum_{i=1}^{n}\left(M_{i R}^{2} / r_{i}\right)}-1}{\sqrt{1+\left(\omega_{0}^{2} / r_{R}\right) \sum_{i=1}^{n}\left(M_{i R}^{2} / r_{i}\right)}+1} .
\end{aligned}
$$

We set

$$
A_{n}=\sqrt{1+\frac{\omega_{0}^{2}}{r_{R}} \sum_{i=1}^{n} \frac{M_{i R}^{2}}{r_{i}}} .
$$

The expressions of optimal load and transfer efficiency in (12) and (13) can be rewritten as

$$
\begin{aligned}
R_{L, \mathrm{OPT}} & =r_{R} A_{n}, \\
\mathrm{PTE}_{\mathrm{OPT}} & =\frac{R_{L, \mathrm{OPT}}-r_{R}}{R_{L, \mathrm{OPT}}+r_{R}}=\frac{A_{n}-1}{A_{n}+1} .
\end{aligned}
$$

Forms of expression in (15) and (16) for calculating the optimal load and transfer efficiency for the multiple-TX WPT system are identical to the forms for multiple-RX systems presented in reference [15]. It can also be found that both $R_{L, \mathrm{OPT}}$ and PTE $\mathrm{OPPT}_{\mathrm{O}}$ increase with the number of TXs. The reason for this is that the total coupling between the TX and RX increases and the equivalent parallel ESR reduces when the number of TXs increases.

\section{Numerical Calculations and Experiments}

In order to illustrate the analyses in part II, we use MATLAB software to perform the numerical calculations. An important target of using such multiple-TX WPT techniques is to improve the energy efficiency of the system. Thus, in the designed system, multiple small TXs with the same size and 


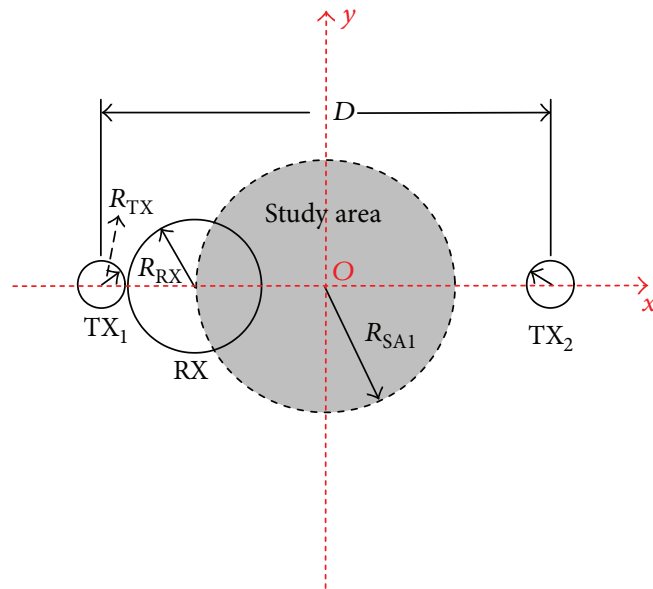

(a)

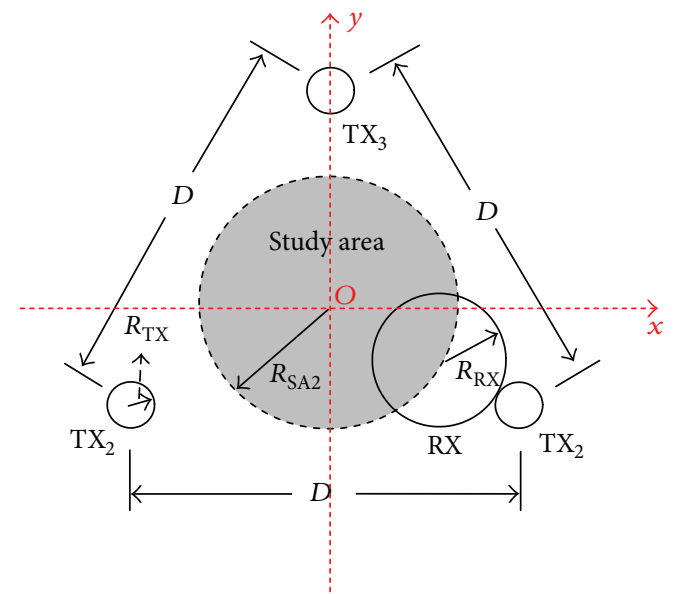

(b)

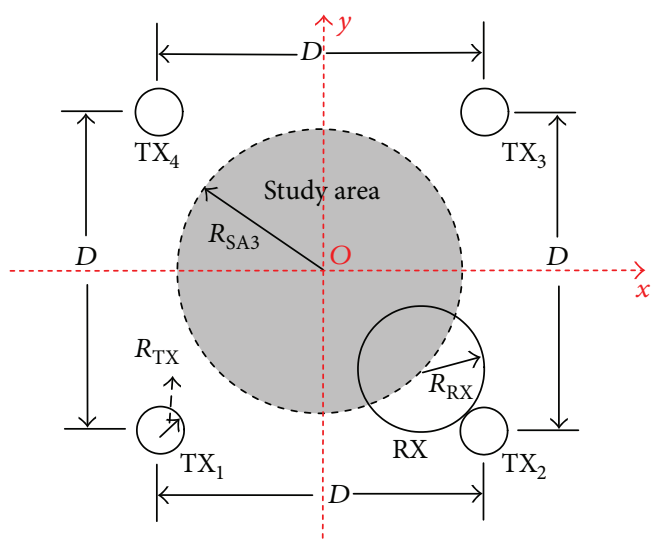

(c)

Figure 3: Spatial layout for a multiple-TX single-RX system: (a) two TX, (b) three TX, and (c) four TX.

a large RX are considered, which is used for decreasing the coupling between TXs to satisfy the theoretical presuppositions. To facilitate comparison discussion for the multipleTX WPT system, coils of TXs are placed side to side with a coil of RX in this part for numerical calculations and experiments. The center of the study area, $o$, is set as the origin of a rectangular coordinate, which is shown with dashed red line in Figure 2. The RX loaded with resistance moves in the study area to investigate the PTE. The center of the RX does not go out of the boundary of the study area. Namely, point $o^{\prime}$ always moves within the grey dotted circle, shown as Figure 2.

The radii of RX and the study area are $R_{\mathrm{RX}}$ and $R_{\mathrm{SA} i}$, respectively, where $i=1,2, \ldots, \mathrm{n}$ for various numbers of TXs. The multiple TXs are distributed evenly around the study area. The system layouts for two, three, and four TXs are shown in Figures 3(a)-3(c), respectively. The radius of each TX is $R_{\mathrm{TX}}$, and the distance between TXs is $D$ for each case. Therefore, the radii of the study area for two-, three-, and four-TX cases are $\quad R_{\mathrm{SA} 1}=D / 2-\left(R_{\mathrm{TX}}+R_{\mathrm{RX}}\right), \quad R_{\mathrm{SA} 2}=D / \sqrt{3}-\left(R_{\mathrm{TX}}+R_{\mathrm{RX}}\right)$ and $R_{\mathrm{SA} 3}=D / \sqrt{2}-\left(R_{\mathrm{TX}}+R_{\mathrm{RX}}\right)$, respectively. It should be clear that $R_{\mathrm{SA} 1}<R_{\mathrm{SA} 2}<R_{\mathrm{SA} 3}$. Table 1 provides a summary of relevant dimensions for the system.
TABLE 1: Dimensions of system structure.

\begin{tabular}{lc}
\hline Dimension & Value \\
\hline$D$ & $1 \mathrm{~m}$ \\
$R_{\mathrm{TX}}$ & $5.5 \mathrm{~cm}$ \\
$R_{\mathrm{RX}}$ & $15.75 \mathrm{~cm}$ \\
$R_{\mathrm{SA} 1}$ & $29 \mathrm{~cm}$ \\
$R_{\mathrm{SA} 2}$ & $36.9 \mathrm{~cm}$ \\
$R_{\mathrm{SA} 3}$ & $49.5 \mathrm{~cm}$ \\
Turn number of TX & 25 \\
Turn number of RX & 11 \\
High of TX & $3.4 \mathrm{~cm}$ \\
High of RX & $1.5 \mathrm{~cm}$ \\
\hline
\end{tabular}

The general calculation method of mutual inductance between each TX and RX has been presented in the former works of our team $[2,8]$. The electrical parameters of TX and RX are listed as follows: parasitic resistances including the ESR of external series capacitor, self-inductance, and capacitance of external series capacitor of TX are $5 \Omega$, 


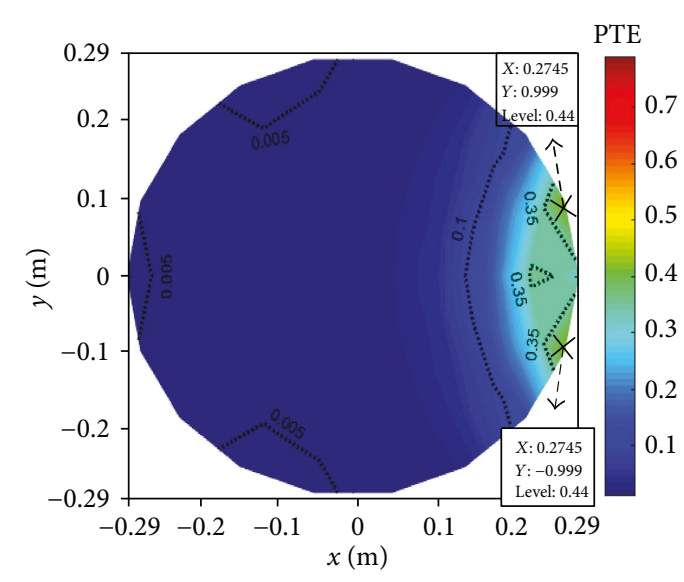

(a)

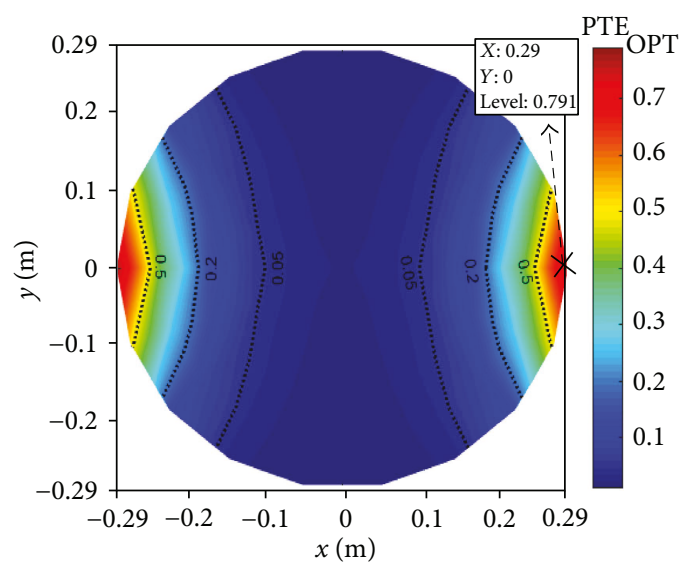

(c)

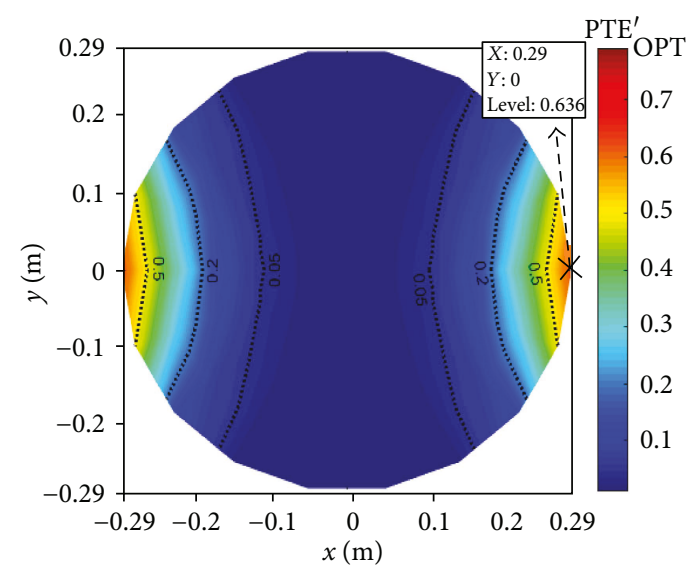

(b)

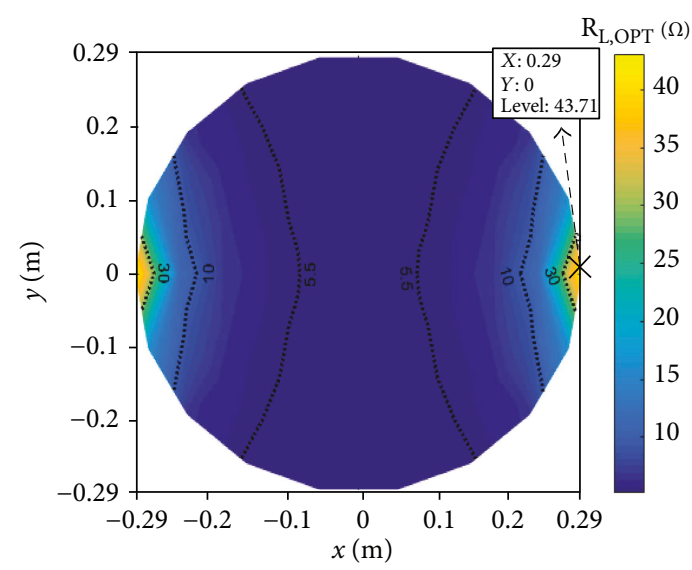

$(\mathrm{d})$

FIgUre 4: Calculated values of a two-TX system when RX is moving in the study area. Efficiency distribution in the study area (a) without optimization, (b) under conditions of $V_{1}: V_{2}=M_{1 R}: M_{2 R}$ and $R_{L}=10 \Omega$, and (c) under conditions of $V_{1}: V_{2}=M_{1 R}: M_{2 R}$ and $R_{L}=R_{L, \mathrm{OPT}} \cdot(\mathrm{d})$ Optimal load for $\mathrm{PTE}_{\mathrm{OPT}}$.

$198.6 \mu \mathrm{H}$, and $110.8 \mathrm{pF}$, respectively, and the counterparts of RX are $5.1 \Omega, 132.8 \mu \mathrm{H}$, and $165.7 \mathrm{pF}$, respectively. The capacitance values of capacitors are selected to make the TXs and RX resonate at the same frequency of $1.073 \mathrm{MHz}$.

Figure 4(a) shows the efficiency in the circular study area under a fixed voltage ratio and load, $V_{1}: V_{2}=1: 5$ and $R_{L}=10 \Omega$. It can be found find that the three maximum efficiency regions occur around $T X_{2}$, which is fed by a high-voltage amplitude. It is noticed that the efficiency increases nonmonotonously with a decreasing distance between RX and $\mathrm{TX}_{2}$. PTE $\mathrm{P}_{\mathrm{OPT}}^{\prime}$ obtained under conditions of $V_{1}: V_{2}=M_{1 R}: M_{2 R}$ and $R_{L}=10 \Omega$ is shown in Figure 4(b). Comparing with the efficiency without any optimal operation shown in Figure 4(a), the efficiency in this case is obviously higher, and maximum values are close to TXs. Under conditions of $V_{1}: V_{2}=M_{1 R}: M_{2 R}$ and $R_{L}=R_{L, \mathrm{OPT}}$ shown in Figure $4(\mathrm{~d})$, the optimal efficiency of $\mathrm{PTE}_{\mathrm{OPT}}$ is plotted in Figure 4(c). It can be observed that the efficiency is further improved comparing with Figure 4(b).

Loading with $R_{L}=10 \Omega$, the nonoptimized efficiencies for the three-TX system under $V_{1}: V_{2}: V_{3}=1: 5: 10$ and the
four-TX case under $V_{1}: V_{2}: V_{3}: V_{4}=1: 5: 10: 20$ are presented in Figure 5(a) and Figure 6(a), respectively. The maximum efficiency appears close to the TX fed with the highest voltage amplitude and towards the TX with the second-highest voltage amplitude, such as the position of $(0.058,0.33)$ for maximum $\mathrm{PTE}=0.26$ close to $\mathrm{TX}_{3}$ and towards $\mathrm{TX}_{2}$ in the three-TX system. Similarly, the position of $(-0.25,0.43)$ for maximum PTE $=0.24$ is close to $\mathrm{TX}_{4}$ and towards $\mathrm{TX}_{3}$ and $(0.23,0.39)$ for the secondary maximum $\mathrm{PTE}=0.04$ is close to $\mathrm{TX}_{3}$ and towards $\mathrm{TX}_{4}$ in the four-TX case.

In the condition of $V_{1}: V_{2}: V_{3}=M_{1 R}: M_{2 R}: M_{3 R}$, Figure 5(b) plots the optimal efficiency $\mathrm{PTE}_{\mathrm{OPT}}^{\prime}$ under $R_{L}=10 \Omega$ and Figure 5(c) plots the final optimal efficiency PTE $_{\mathrm{OPT}}$ under the optimal load $R_{L, \mathrm{OPT}}$ shown in Figure $5(\mathrm{~d})$ in the three-TX system. Similarly, in the fourTX case, when $V_{1}: V_{2}: V_{3}: V_{4}=M_{1 R}: M_{2 R}: M_{3 R}: M_{4 R}$, Figure 6(b) plots PTE ${ }_{\mathrm{OPT}}^{\prime}$ under $R_{L}=10 \Omega$ and Figure 6(c) plots $\mathrm{PTE}_{\mathrm{OPT}}$ under $R_{L}=R_{L, \mathrm{OPT}}$ shown in Figure $6(\mathrm{~d})$. As a result, $\mathrm{PTE}_{\mathrm{OPT}}>\mathrm{PTE}_{\mathrm{OPT}}^{\prime}>\mathrm{PTE}$ in each case when $\mathrm{RX}$ moves in the circle of the study area. All of the maximum $\mathrm{PTE}_{\mathrm{OPT}} \mathrm{s}$ 


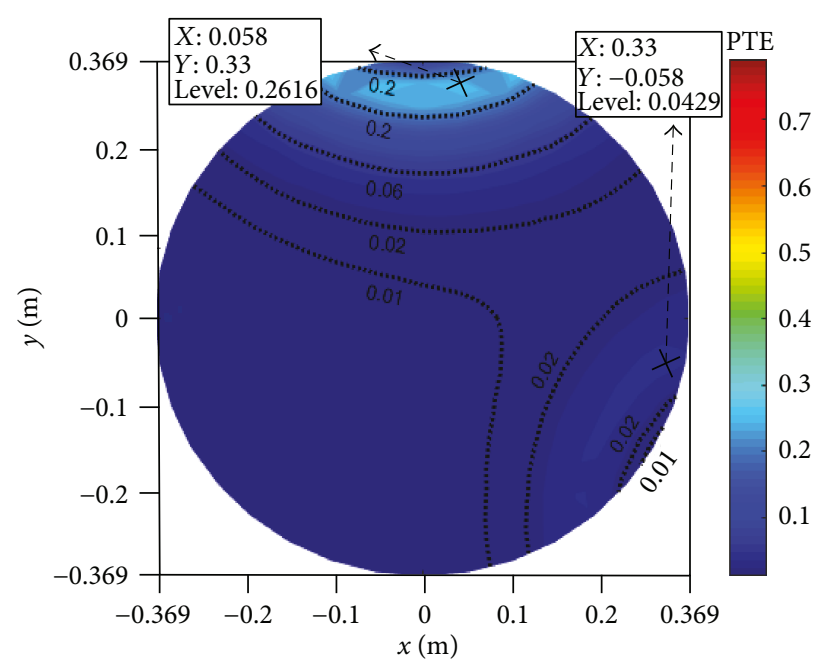

(a)

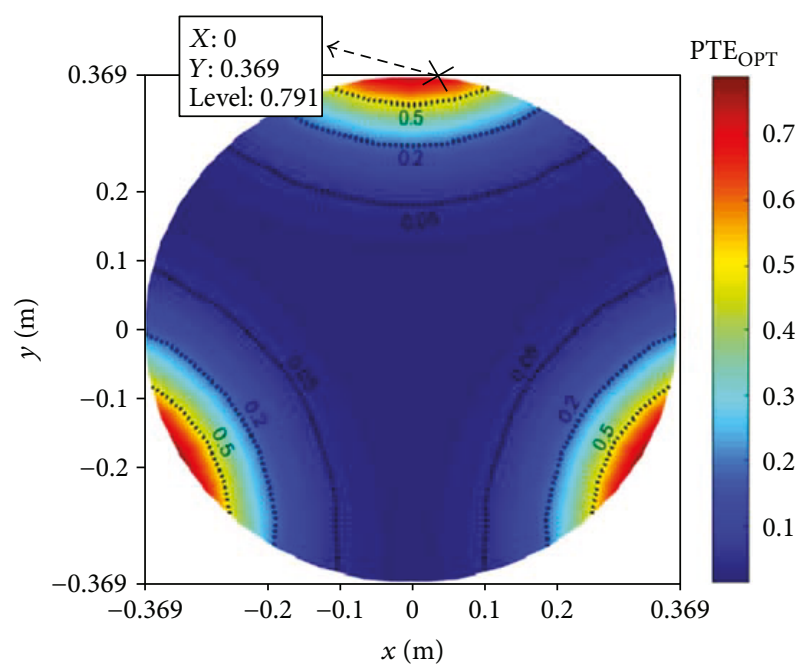

(c)

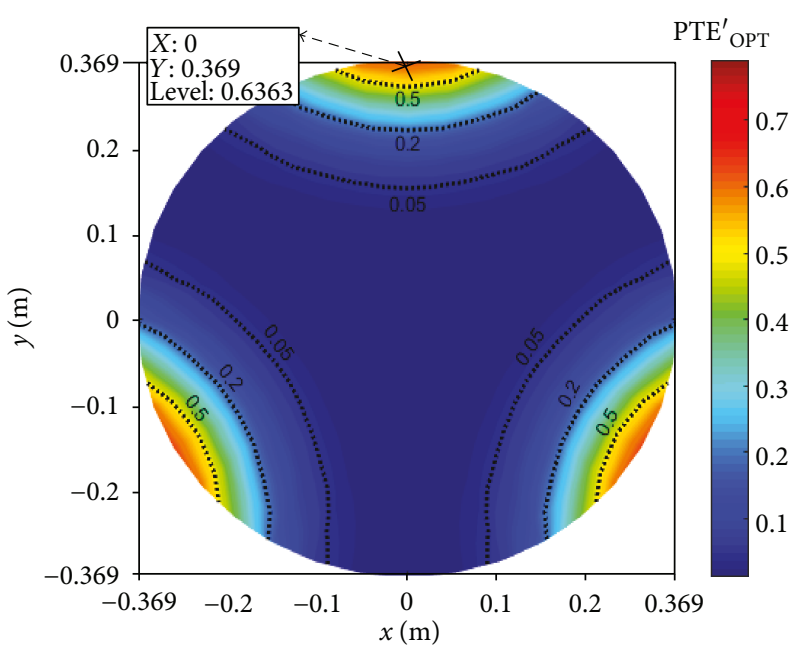

(b)

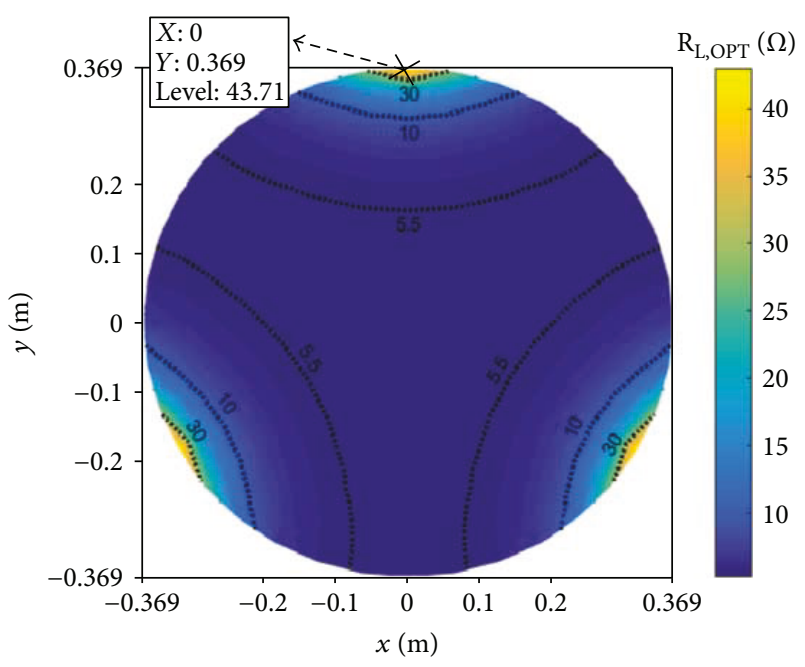

(d)

Figure 5: Calculated values of a three-TX system when RX is moving in the study area. Efficiency distribution in the study area (a) without optimization, (b) under conditions of $V_{1}: V_{2}: V_{3}=M_{1 R}: M_{2 R}: M_{3 R}$ and $R_{L}=10 \Omega$, and (c) under conditions of $V_{1}: V_{2}: V_{3}=M_{1 R}: M_{2 R}: M_{3 R}$ and $R_{L}=R_{L, \mathrm{OPT}}$. (d) Optimal load for $\mathrm{PTE}_{\mathrm{OPT}}$.

of two-, three-, and four-TX systems are 0.79 when the RX is $R_{\mathrm{TX}}+R_{\mathrm{RX}}$ apart from any one of the TXs. It shows that the maximum efficiency mainly depends on the mutual inductance between the nearest TX and the RX when the system satisfies the two optimization conditions. Comparing with the multiple-TX systems, we can find that the effective charging areas increase with the increasing number of TXs. Thus, using a multiple-TX system can maximize the flexibility of the WPT application.

From the investigations of the optimal PTE in Figures 4, 5 , and 6 for two-, three-, and four-TX systems, respectively, two problems need to be illuminated:

(1) The maximum values of optimal PTE in the three kinds of multiple-TX systems are the same result of 0.791 and always appear at the closest position to the TXs. Similarly, the optimal load values for the maximum PTE are also the same. These are due to the distance between TXs $D=1 \mathrm{~m}$, which is large enough that the mutual coupling effect of the nearest TX is much higher than those of the other TXs. The mutual inductance between RX and the nearest TX is almost five times the inductance between $\mathrm{RX}$ and the other TXs.

(2) Two parameters of PTE and power delivered to the load (PDL) are the main criterions to evaluate the performance of a WPT system. The high PTE means a low system loss; however, if feeding power is largely limited under optimal PTE conditions, the PDL of this system would be very low. Here, we present the PDL of a multiple-TX system under the optimal PTE conditions. The specific values of PDL can be calculated with specific values of 


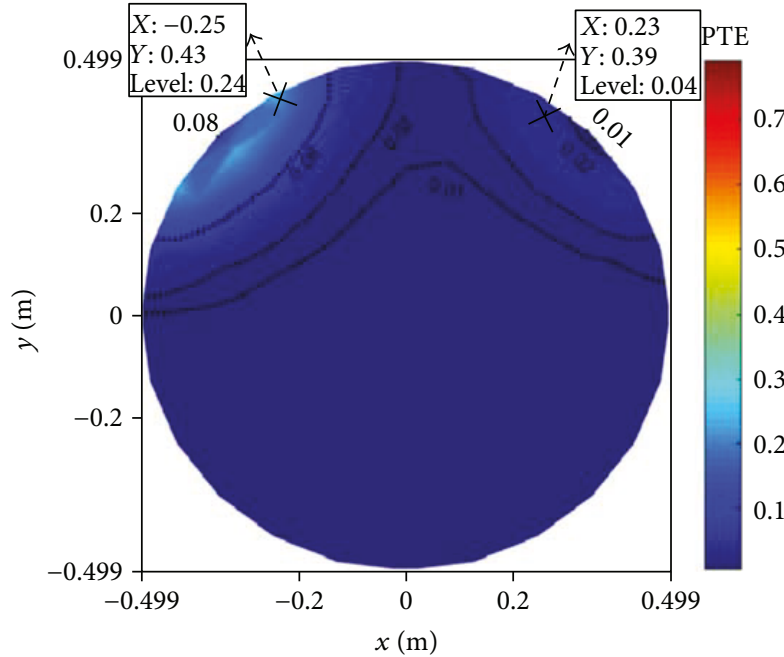

(a)

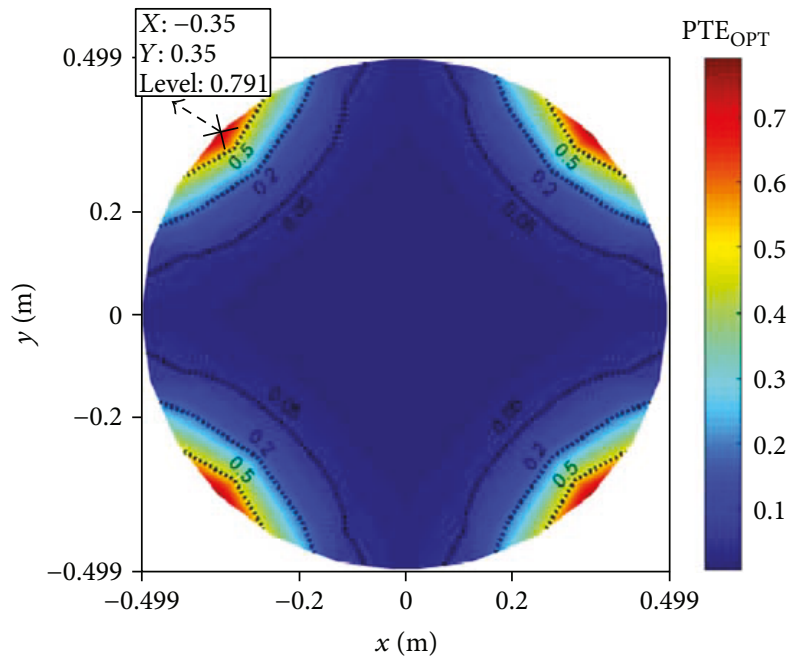

(c)

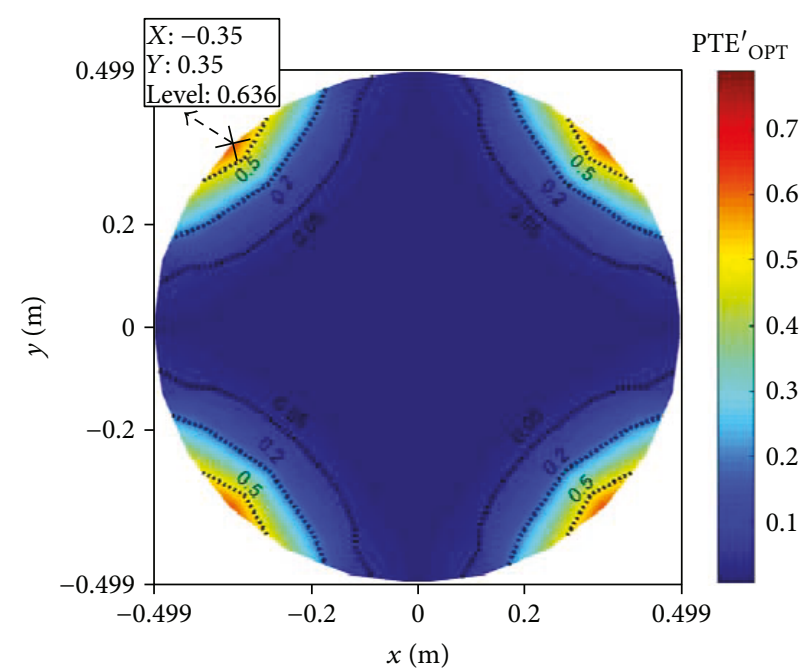

(b)

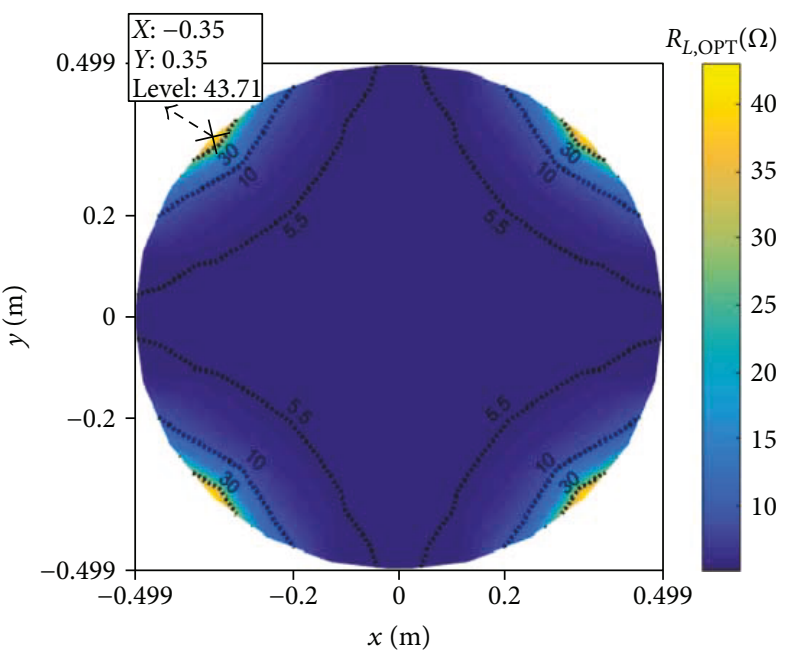

(d)

FIgURE 6: Calculated values of a four-TX system when RX is moving in the study area. Efficiency distribution in the study area (a) without optimization, (b) under conditions of $V_{1}: V_{2}: V_{3}: V_{4}=M_{1 R}: M_{2 R}: M_{3 R}: M_{4 R}$ and $R_{L}=10 \Omega$, and (c) under conditions of $V_{1}: V_{2}: V_{3}$ $: V_{4}=M_{1 R}: M_{2 R}: M_{3 R}: M_{4 R}$ and $R_{L}=R_{L, \mathrm{OPT}}$ (d) Optimal load for PTE $\mathrm{OPPT}_{\mathrm{O}}$.

feeding voltage. Therefore, setting up reference values of $M_{0 R}=10^{-5} \mathrm{H}$ and $V_{0}=1 \mathrm{~V}, V_{i, \mathrm{PTE}}(i=1, \ldots, n)$ can be obtained according to $V_{0}: V_{1}: \cdots: V_{n}=$ $M_{0 R}: M_{1 R}: \cdots: M_{n R}$ and the calculated mutual inductance value of $M_{j R}(j=0, \ldots, n)$ for a different receive location of RX. The calculated feeding voltage values of $\mathrm{TX}_{1}$ for two-, three-, and four-TX systems versus the position of $\mathrm{RX}$ are shown in Figure 7. The other feeding voltage values of $\mathrm{TX}_{k}$ $(k=2, \ldots, n)$ are circular symmetric with those of $\mathrm{TX}_{1}$ about the center of the study area. Using the feeding voltage in Figure 7 and optimal load in Figures 4(d), 5(d), and 6(d), namely, under optimal PTE conditions, Figure 8 gives the PDL for two-, three-, and four-TX systems. It can be found that the maximum $\mathrm{PDL}$ is $0.26 \mathrm{~W}$ using maximum feeding voltage value of $0.74 \mathrm{~V}$. This power level of PDL is sufficient for the charging of consumer electronics and the nodes in internet of things, and so on, through proper adjustment of feeding voltage amplitudes. Therefore, the studies in this paper are significant. Similarly, the maximum feeding voltage and PDL for two-, three-, and four-TX systems are the same due to the long distances between TXs selected in this paper.

According to the theoretical analysis presenting in Section 2, we manufacture five TXs and one RX with the sizes shown in the numerical calculation part to measure the $\mathrm{PTE}_{\mathrm{OPT}}$. To ensure each TX with the predetermined amplitude and phase of feeding voltage, a lumped transformer with a Mn-Zn-type toroid ferrite core is used to 


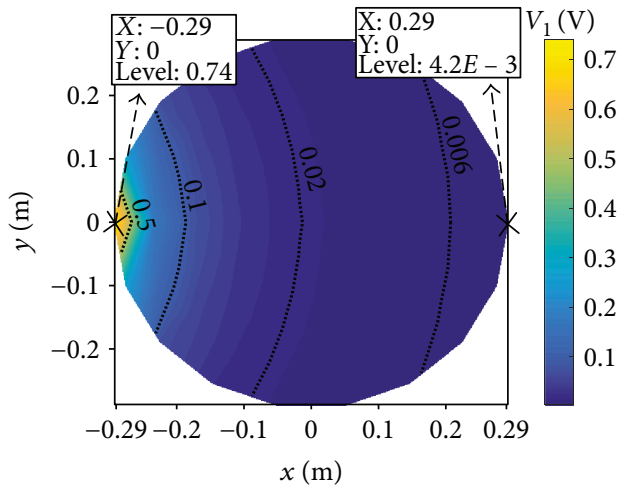

(a)

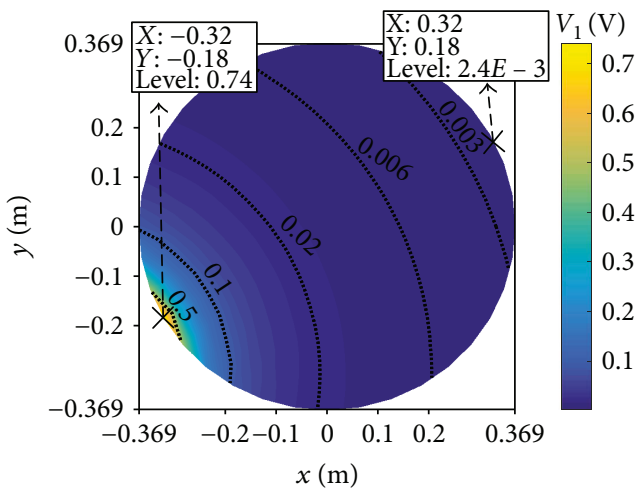

(b)

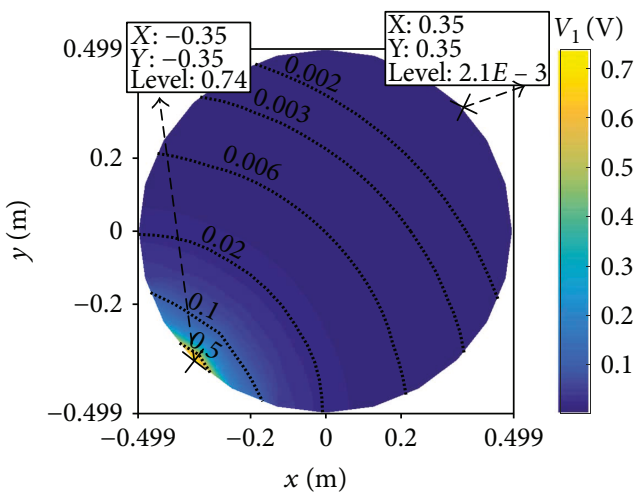

(c)

FIgURe 7: The voltage values of $\mathrm{TX}_{1}$ for calculating PDL under conditions of maximizing PTE: (a) two-, (b) three-, and (c) fourTX systems.

deliver the power of one channel coming from the source into several channels feeding to multiple TXs. Each TX is set at the same distance away from the RX, and then the amplitudes of the feeding voltage for at most five TXs are set to identical values to obtain optimal efficiency. As shown in the subgraph in Figure 9(a), the lumped transformer consists of six groups of winding on a ferrite core for a five-TX system. The turn number of each group of winding is the same, 3 for this experiment, and one group of winding connects the function generator and the others feed the identical power to the multiple TXs.

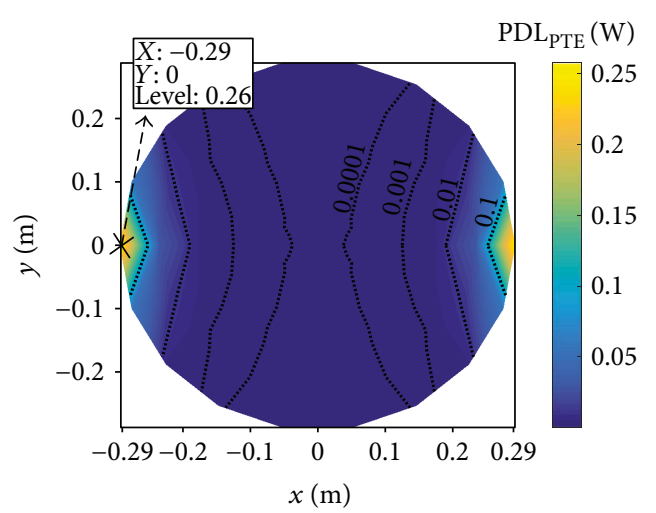

(a)

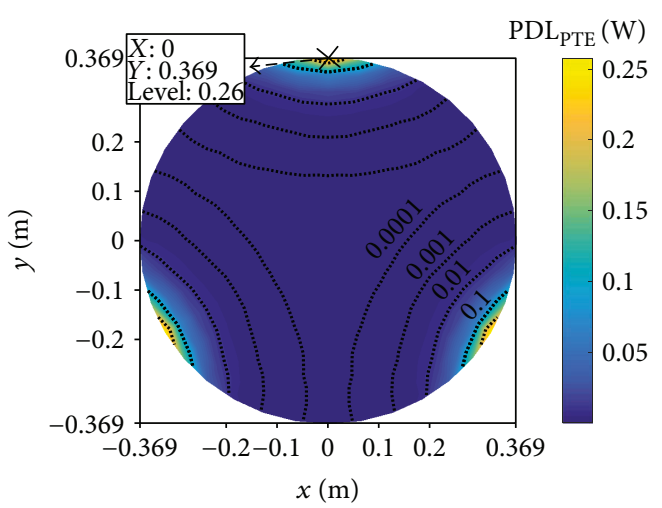

(b)

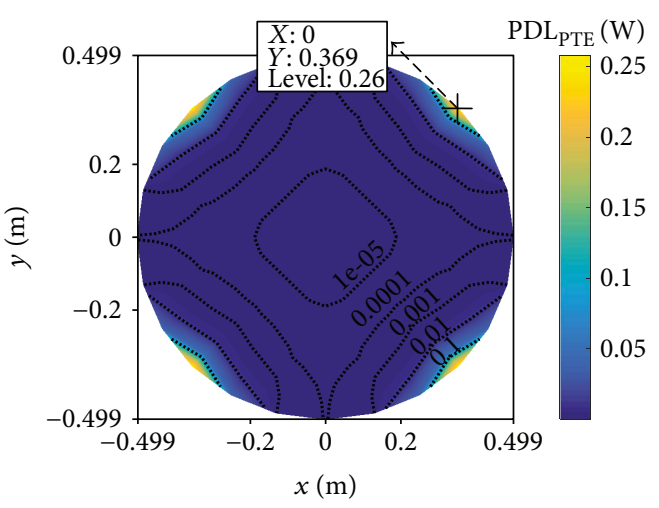

(c)

FIGURE 8: The values of PDL under conditions of maximizing PTE: (a) two-, (b) three-, and (c) four-TX systems.

Figure 9(a) shows the overall experiment setup for the five-TX case.

Figure 9(b) shows the calculated and experimental values of $\mathrm{PTE}_{\mathrm{OPT}}$ and the optimal load for PTE $\mathrm{OPT}_{\mathrm{OPT}}$ versus the number of TXs when $D_{i R}=0.26 \mathrm{~m}(i=1,2, \ldots, 5)$, corresponding to $M_{i R}=2.17 \mu \mathrm{H}$, where $D_{i R}$ is the distance between the RX and each one of the TXs. The calculated $\mathrm{PTE}_{\mathrm{OPT}}$ and $R_{L, \mathrm{OPT}}$ increases with increasing number of TXs, which is consistent with the analysis in Section 2. The experimental results of $\mathrm{PTE}_{\mathrm{OPT}}^{\prime}$ of at most a fiveTX system shown in circular dots match well with the calculated results. 


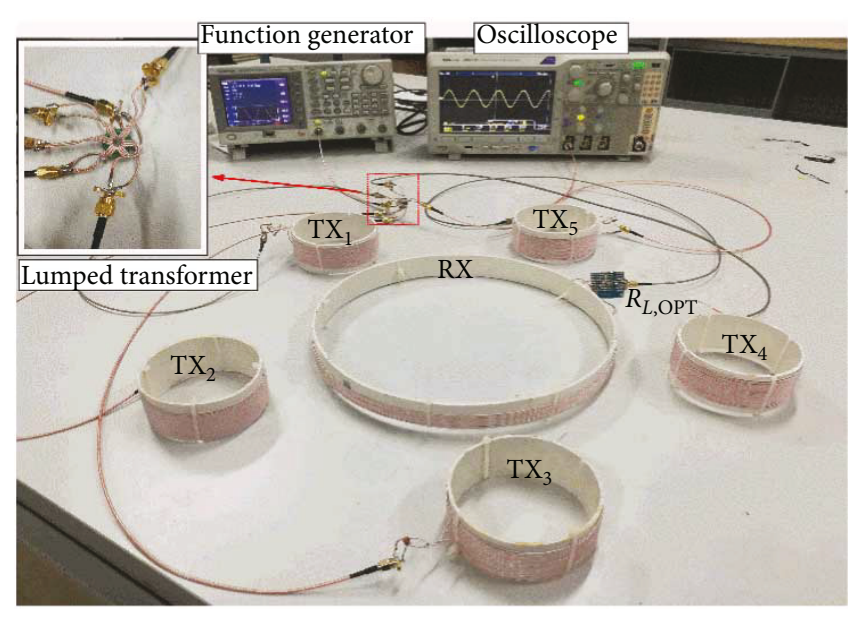

(a)

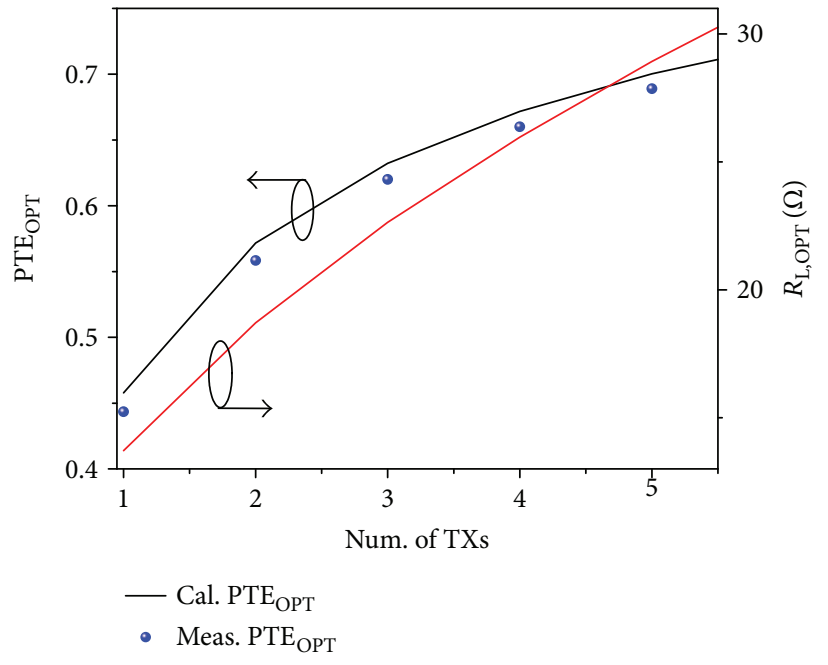

(b)

FIGURE 9: Experimental investigation of PTE $\mathrm{OPT}_{\text {versus the number of TXs: (a) example of experimental setup; (b) optimal load for PTE }}$ OPT and measured values of $\mathrm{PTE}_{\mathrm{OPT}}$ comparing with the calculated data versus the number of TXs.

To further confirm the conclusion obtained from analysis, two TXs and one RX are arranged face to face to construct a complete system, and the distance between the two TXs is fixed at $0.6 \mathrm{~m}$. Using the dual channel function generator shown in Figure 9(a), the amplitude ratio and phase difference of two feeding voltages can be adjusted to arbitrary values. For comparison, two PTEs of voltage ratios of $V_{1}: V_{2}=1: 1$ and $1: 3$ with $R_{L}=50 \Omega$ are investigated. Calculated and measured efficiencies with and without the optimal conditions are compared in Figure 10(b) when the distance between $\mathrm{RX}$ and $\mathrm{TX}_{1}, D_{1 R}$, changes from $0.05 \mathrm{~m}$ to $0.55 \mathrm{~m}$. The optimal mutual inductance ratios and loads for $\mathrm{PTE}_{\mathrm{OPT}}$ and $\mathrm{PTE}_{\mathrm{OPT}}^{\prime}$ are plotted in Figure 10(a). It can be found that $P T E=P T E_{O P T}^{\prime}$ at $D_{1 R}=0.3 \mathrm{~m}$ and $0.37 \mathrm{~m}$ for $V_{1}: V_{2}=1: 1$ and $1: 3$, respectively. The reason for these is that $M_{1 R}: M_{2 R}=1$ and 0.33 as shown in Figure 10(a), which satisfies the optimal condition of $V_{1}: V_{2}=M_{1 R}: M_{2 R}$ to maximize the PTE with an unoptimized load. As a result, the $\mathrm{PTE}_{\mathrm{OPT}}$ shown in black line in Figure 10(b) under the conditions of $V_{1}: V_{2}=M_{1 R}: M_{2 R}$ and $R_{L}=R_{L, \mathrm{OPT}}$ has the highest values. Duo to the influence of a strong paramagnetic response [2, 19], the measured values for the scenario of the fixed voltage ratio and load slightly deviate from the calculated values when RX gets close to TX. But overall, the measurements for different scenarios agree with the calculated values.

In this section, we also present the PTE characteristics of a one-TX and one-RX system. The values of PTE of a one-TX system with an optimal load and a fixed load of $50 \Omega$ are plotted in Figure 10. It is clear that the optimal PTE of the two-TX system is slightly larger than that of the one-TX system when $\mathrm{RX}$ is close to $T X_{1}$ since $M_{1 R}^{2} / r_{1}$ for the
one-TX system is almost equal to $\sum_{i=1}^{2}\left(M_{i R}^{2} / r_{i}\right)$ for the two-TX system in this case; however, when RX is close to $\mathrm{TX}_{2}, M_{1 R}^{2} / r_{1}$ is much less than $\sum_{i=1}^{2}\left(M_{i R}^{2} / r_{i}\right)$ which results in the value of PTE for the one-TX system being far below that for the two-TX system.

\section{Conclusions}

In this paper, the procedures of efficiencies for a multiple-TX WPT system are analyzed in details based on the circuit model. There are two steps to obtain the highest efficiency via the theoretical analysis. Firstly, the feeding voltage ratio should be set to the mutual inductance ratio to achieve a higher PTE. Then the optimal load needs be attached to the RX to obtain the highest efficiency. The expressions for the highest efficiency and optimal loads for any multiple-TX system can be unified to the same form. Numerical results of two-, three-, and four-TX systems are further illustrated to compare the optimal and nonoptimized efficiencies. Experiments for a two-TX system are carried out to verify the numerical analysis when transmission distance is changed. Theoretical and experimental results indicate that the optimal efficiency increases along with the number of TXs mounting up.

In terms of two optimal conditions for the highest efficiency, the expressions of the conditions are simple but universal, which provide a guideline for the optimal design of a multiple-TX WPT system.

The proposed multiple-TX system is easy to fit on the surrounding environment (such as four TXs embedded in the four corners of a table and one RX 


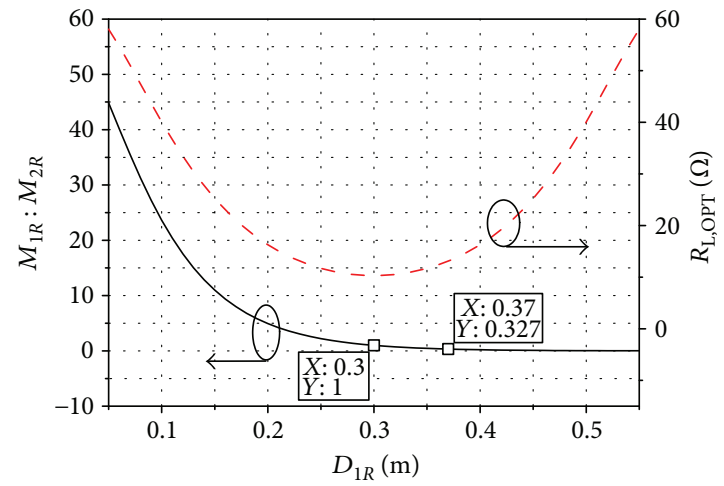

(a)

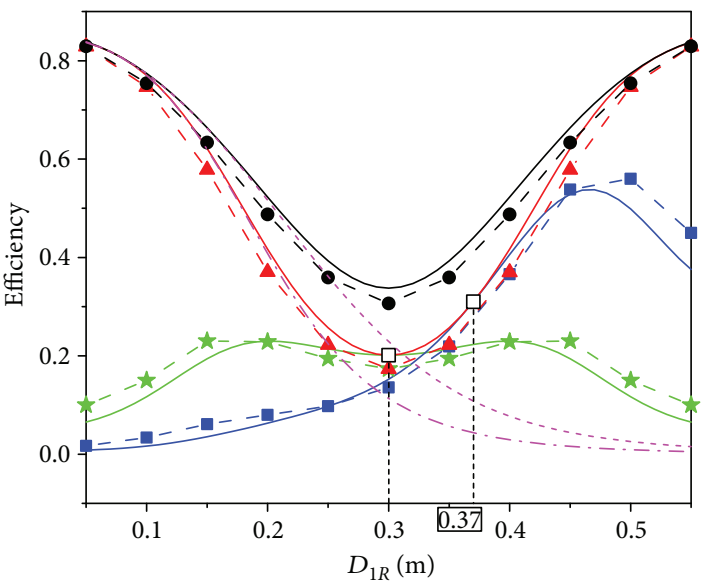

$$
\begin{aligned}
& \text { One-TX system: } \\
& \text {-.. Cal. PTE } R_{L}=50 \\
& \text {--- Cal. PTE }{ }_{\mathrm{OPT}} R_{L}=R_{L, \mathrm{OPT}} \\
& \text { Two-TX system: } \\
& \text { C Cal. PTE } V_{1}: V_{2}=1: 1 ; R_{L}=50 \\
& \text { - } \star \text { - Meas. PTE } V_{1}: V_{2}=1: 1 ; R_{L}=50 \\
& \text { Cal. PTE } V_{1}: V_{2}=1: 3 ; R_{L}=50 \\
& \text { - - Meas. PTE } V_{1}: V_{2}=1: 3 ; R_{L}=50 \\
& \text { — Cal. PTE }{ }_{\mathrm{OPT}}^{\prime} V_{1}: V_{2}=M_{1 R}: M_{2 R} ; R_{L}=50 \\
& \text { - } \Delta \text { - Meas. } \mathrm{PTE}_{\mathrm{OPT}}^{\prime} V_{1}: V_{2}=M_{1 R}: M_{2 R} ; R_{L}=50 \\
& \text { - Cal. } \mathrm{PTE}_{\mathrm{OPT}}^{\prime} V_{1}: V_{2}=M_{1 R}: M_{2 R} ; R_{L}=R_{L, \mathrm{OPT}} \\
& \text {-•- Meas. PTE }{ }_{\mathrm{OPT}}^{\prime} V_{1}: V_{2}=M_{1 R}: M_{2 R} ; R_{L}=R_{L, \mathrm{OPT}}
\end{aligned}
$$

(b)

FIGURE 10: Efficiencies versus transfer distance when two TXs and a single RX are arranged face to face to construct a complete system: (a) mutual inductance ratio and optimal load for the highest efficiency; (b) calculated and measured efficiency values under different conditions.

positioned on any point of the table) to achieve a large effective charging area.

\section{Data Availability}

The data that support the findings of this study are available from the corresponding author upon reasonable request.

\section{Conflicts of Interest}

The authors declare that there are no conflicts of interest regarding the publication of this paper.

\section{Acknowledgments}

This research work was supported in part by the Scientific Research Foundation for the High-Level Talents of Jinling Institute of Technology (jit-b-201719), the National Natural Science Foundation of China (61601245), and the Nanjing University of Posts and Telecommunications Foundation of China (Grant no. NY 214050).

\section{References}

[1] A. Kurs, A. Karalis, R. Moffatt, J. D. Joannopoulos, P. Fisher, and M. Soljačić, "Wireless power transfer via strongly coupled magnetic resonances," Science, vol. 317, no. 5834, pp. 8386, 2007. 
[2] J. Zhang and C. Cheng, "Analysis and optimization of threeresonator wireless power transfer system for predeterminedgoals wireless power transmission," Energies, vol. 9, no. 4, p. 274, 2016.

[3] Y. Zhang, Z. Zhao, and K. Chen, "Frequency-splitting analysis of four-coil resonant wireless power transfer," IEEE Transactions on Industry Applications, vol. 50, no. 4, pp. 24362445, 2014.

[4] Y. L. Lyu, F. Y. Meng, G. H. Yang et al., "A method of using nonidentical resonant coils for frequency splitting elimination in wireless power transfer," IEEE Transactions on Power Electronics, vol. 30, no. 11, pp. 6097-6107, 2015.

[5] W.-S. Lee, W.-I. Son, K.-S. Oh, and J.-W. Yu, "Contactless energy transfer systems using antiparallel resonant loops," IEEE Transactions on Industrial Electronics, vol. 60, no. 1, pp. 350-359, 2013.

[6] X. Y. Zhang, C. D. Xue, and J. K. Lin, "Distance-insensitive wireless power transfer using mixed electric and magnetic coupling for frequency splitting suppression," IEEE Transactions on Microwave Theory and Techniques, vol. 65, no. 11, pp. 4307-4316, 2017.

[7] M. Kiani and M. Ghovanloo, "The circuit theory behind coupled-mode magnetic resonance-based wireless power transmission," IEEE Transactions on Circuits and Systems I: Regular Papers, vol. 59, no. 9, pp. 2065-2074, 2012.

[8] C. Cheng and J. Zhang, "Comparative studies between KVL and BPFT in magnetically-coupled resonant wireless power transfer," IET Power Electronics, vol. 9, no. 10, pp. 21212129, 2016.

[9] H. Li, K. Wang, L. Huang, W. Chen, and X. Yang, "Dynamic modeling based on coupled modes for wireless power transfer systems," IEEE Transactions on Power Electronics, vol. 30, no. 11, pp. 6245-6253, 2015.

[10] J. Zhang and C. Cheng, "Quantitative investigation into the use of resonant magneto-inductive links for efficient wireless power transfer," IET Microwaves, Antennas \& Propagation, vol. 10, no. 1, pp. 38-44, 2016.

[11] B. H. Choi and J. H. Lee, "Design of asymmetrical relay resonators for maximum efficiency of wireless power transfer," International Journal of Antennas and Propagation, vol. 2016, Article ID 8247476, 8 pages, 2016.

[12] D.-W. Seo and J.-H. Lee, "Method for estimating optimum free resonant frequencies in overcoupled WPT system," International Journal of Antennas and Propagation, vol. 2017, Article ID 1830687, 6 pages, 2017.

[13] L. Sun, H. Tang, and S. Zhong, "Load-independent output voltage analysis of multiple-receiver wireless power transfer system," IEEE Antennas and Wireless Propagation Letters, vol. 15, pp. 1238-1241, 2016.

[14] X. Huang, J. Guo, F. Wen, and L. Tan, “Output power stabilisation of wireless power transfer system with multiple transmitters," IET Power Electronics, vol. 9, no. 7, pp. 13741380, 2016.

[15] M. Fu, T. Zhang, C. Ma, and X. Zhu, "Efficiency and optimal loads analysis for multiple-receiver wireless power transfer systems," IEEE Transactions on Microwave Theory and Techniques, vol. 63, no. 3, pp. 801-812, 2015.

[16] C. Zhong, B. Luo, F. Ning, and W. Liu, "Reactance compensation method to eliminate cross coupling for two-receiver wireless power transfer system," IEICE Electronics Express, vol. 12, no. 7, article 20150016, 2015.
[17] R. Johari, J. V. Krogmeier, and D. J. Love, “Analysis and practical considerations in implementing multiple transmitters for wireless power transfer via coupled magnetic resonance," IEEE Transactions on Industrial Electronics, vol. 61, no. 4, pp. 17741783, 2014.

[18] H. Ku and P. Kong, "Efficiency optimising scheme for wireless power transfer system with two transmitters," Electronics Letters, vol. 52, no. 4, pp. 310-312, 2016.

[19] D. Ahn, M. Kiani, and M. Ghovanloo, "Enhanced wireless power transmission using strong paramagnetic response," IEEE Transactions on Magnetics, vol. 50, no. 3, pp. 96103, 2014. 


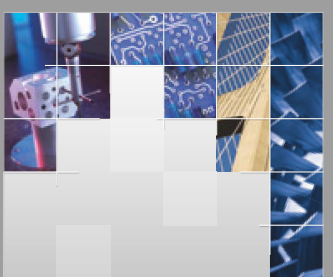

\section{Enfincering}
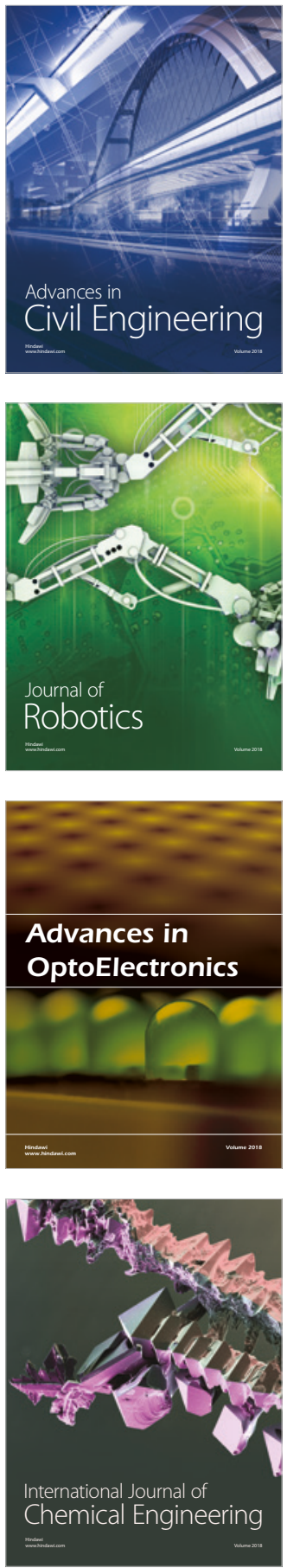

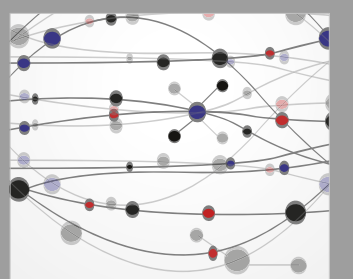

\section{Rotating \\ Machinery}

The Scientific World Journal

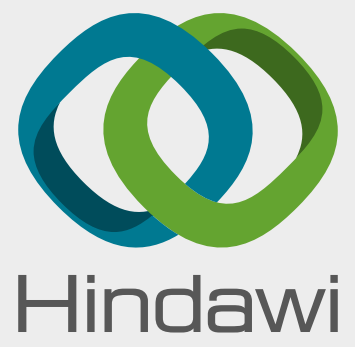

Submit your manuscripts at

www.hindawi.com
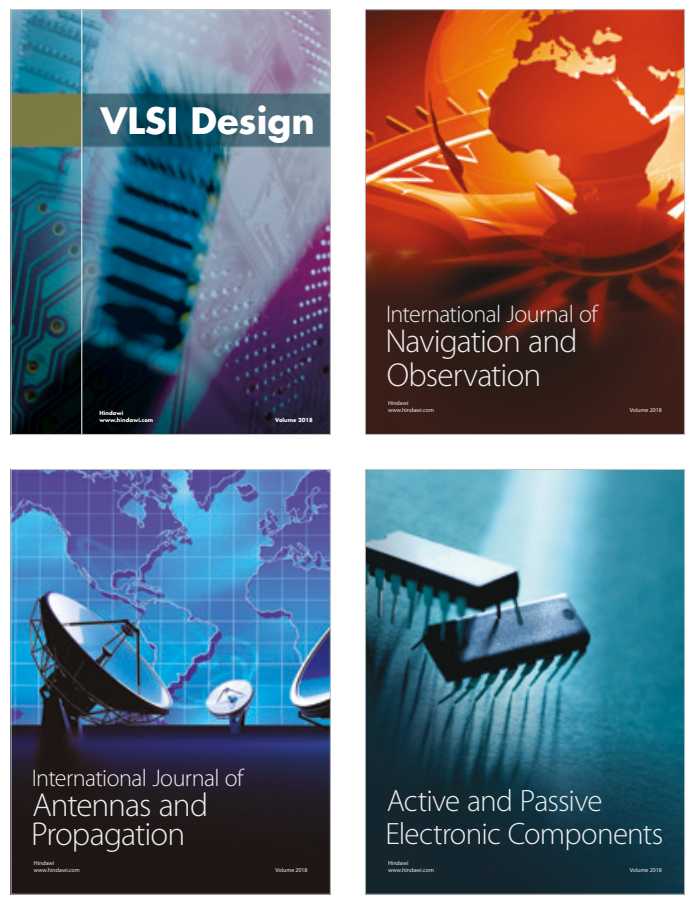
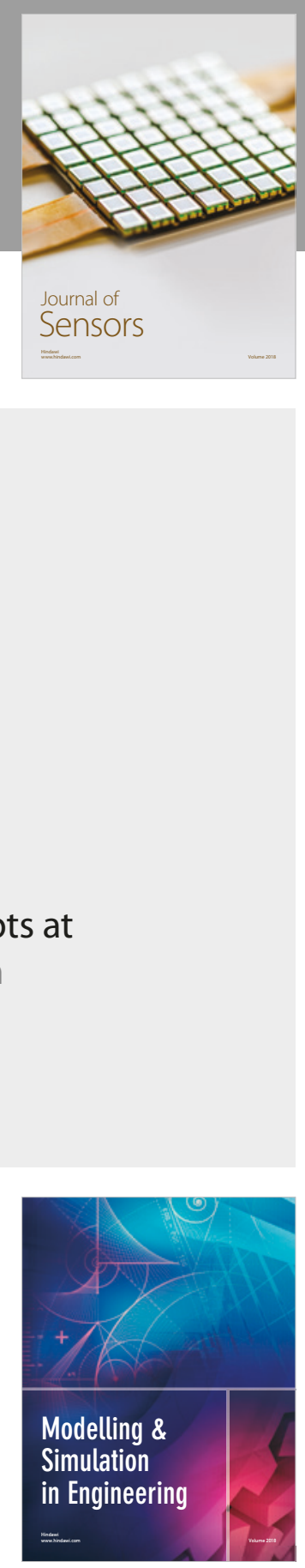

\section{Advances \\ Multimedia}
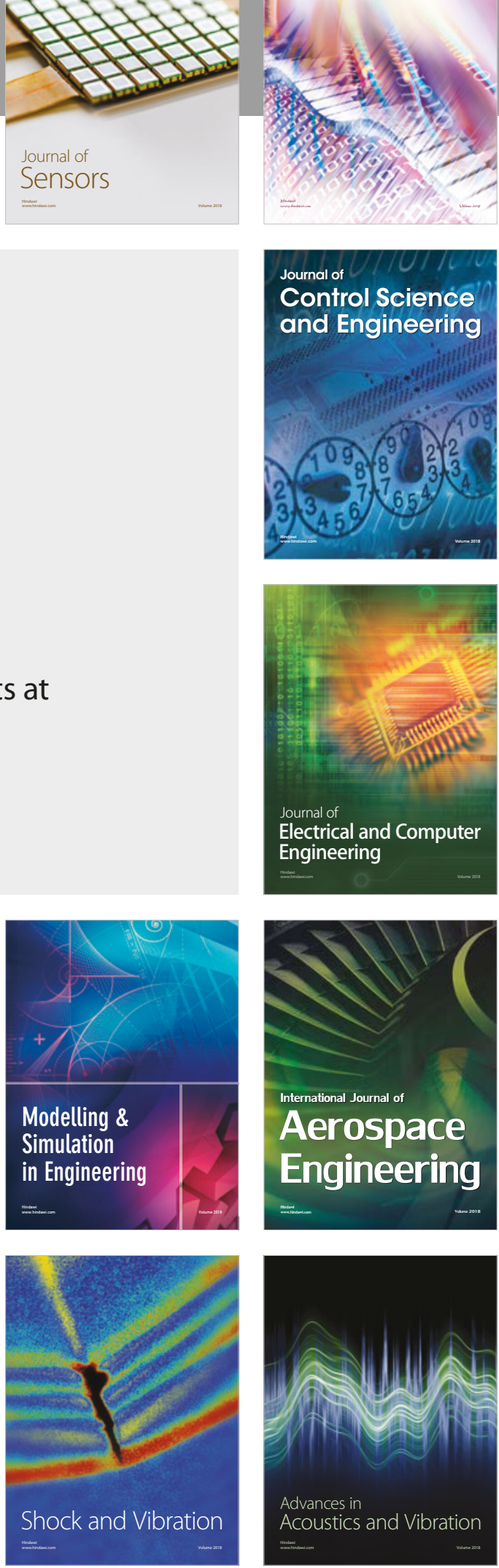